\title{
Capsaicin lindert Gonarthroseschmerzen
}

Stevens RM et al. Randomized, Double-Blind,

Placebo-Controlled Trial of Intraarticular

Trans-Capsaicin for Pain Associated With

Osteoarthritis of the Knee. Arthritis Rheumatol

2019; 71: 1524-1533. doi:10.1002/art.40894

Capsaicin, der „Scharfmacher" in Chilischoten, wird in einer topischen Formulierung zur Behandlung der Zosterneuralgie eingesetzt. Profitieren Patienten mit chronischen, mäßig bis stark ausgeprägten arthrosebedingten Kniegelenkschmerzen von einer einmaligen intraartikulären Capsaicin-Injektion? Diese Frage wollten US-Wissenschaftler im Rahmen einer randomisierten Phase llbStudie klären.

Die Schärfe von Chilischoten beruht auf der Wirkung von Capsaicin, erläutern die Forscher: Die Substanz fungiert als Agonist an einem auf nozizeptiven Nervenendigungen exprimierten Kationenkanal und vermittelt auf diese Weise Schmerzreize. Nach der kurzen Aktivierung der Nozizeptoren setzt jedoch eine Desensibilisierung ein, die über mehrere Wochen - bis zur Regeneration der Rezeptoren - anhält. Die Wissenschaftler testeten nun eigens zur intraartikulären Injektion entwickeltes hochreines TransCapsaicin (CNTX-4975) an einem Kollektiv von 175 Gonarthrosepatienten im Alter zwischen 45 und 80 Jahren, die über chronische, mäßig bis stark ausgeprägte Gelenkschmerzen klagten. Voraussetzung waren stabile Beschwerden über mindestens 2 Monate sowie ein unzureichendes Ansprechen bzw. eine Unverträglichkeit oder Kontraindikationen gegenüber den Standardmedikamenten (nichtsteroidale Antiphlogistika, Opioide, intraartikuläre Glukokortikoide, intraartikuläre Hyaluronsäure). Eine topische Capsaicin-Vorbehandlung, vorangegangene Injektionsbehandlungen sowie chirurgische Eingriffe stellten Ausschlusskriterien dar. Im Rahmen der Dosisfindungsstudie erhielten 34 Patienten einmalig eine CNTX-4975-Injektion in einer Dosis von $0,5 \mathrm{mg}, 71$ Patienten erhielten das Capsaicin-Präparat in einer Dosis von $1,0 \mathrm{mg}$ und 70 eine Placeboinjektion. Im Verlauf der folgenden 24 Wochen stellten sich die Studienteilnehmer regelmäßig zu 
Nachuntersuchungen vor. Studienendpunkte waren die Area under the Curve (AUC) hinsichtlich der prä- zu posttherapeutischen Veränderung des „Western Ontario and McMaster Universities Osteoarthritis Index (WOMAC) pain with walking“ Score nach 12 Wochen. Ferner beurteilten die Forscher die therapeutische Effektivität nach 24 Wochen und erfassten alle auftretenden Nebenwirkungen.

\section{Ergebnisse}

Die Effektivitätsanalyse umfasste $172 \mathrm{~Pa}$ tienten: 33 mit 0,5mg CNTX-4975-, 70 mit 1,0mg CNTX-4975- und 69 mit Placeboinjektion. Bezüglich der Schmerzreduktion nach 12 Wochen erwiesen sich beide CNTX4975-Dosen gegenüber Placebo als überlegen. Die höher dosierte Capsaicin-Formulierung führte dabei zu einer deutlicheren Symptombesserung. Nach 24 Wochen stellten die Wissenschaftler nur noch bei den mit 1,0mg CNTX-4975 behandelten Patienten eine signifikante Schmerzlinderung im Vergleich zu Placebo fest. Neben der analgetischen Wirkung beschrieben die Patienten unter anderem eine Abnahme der Kniesteifigkeit sowie funktionelle Verbesserungen. Bezüglich therapiebedingter $\mathrm{Ne}$ benwirkungen unterschieden sich die 1,0mg CNTX-4975- und die Placebogruppe nicht wesentlich.

\section{FAZIT}

Capsaicin lindert nach intraartikulärer Injektion dosisabhängig arthrosebedingte Knieschmerzen beim Gehen, schlussfolgern die Autoren. Bei höherer Dosierung hält die Symptomlinderung dabei bis zu 24 Wochen an, bei niedriger nur etwa halb so lang. Angesichts der hohen therapeutischen Effektivität und guten Verträglichkeit von CNTX4975 sprechen sich die Studieninitiatoren für eine klinische Weiterentwicklung des Präparats aus.

Dr. med. Judith Lorenz, Künzell 